Proc. Indian Acad. Sci. (Math. Sci.) Vol. 117, No. 2, May 2003, pp. 205-211.

Printed in India

\title{
Borel hierarchies in infinite products of Polish spaces
}

\author{
RANA BARUA and ASHOK MAITRA* \\ Stat-Math Division, Indian Statistical Institute, Kolkata 700 108, India \\ * School of Statistics, University of Minnesota, Minneapolis, MN, USA \\ E-mail: rana@isical.ac.in; maitra@stat.umn.edu \\ MS received 22 August 2005; revised 28 August 2006
}

Abstract. Let $H$ be a product of countably infinite number of copies of an uncountable Polish space $X$. Let $\Sigma_{\xi}\left(\bar{\Sigma}_{\xi}\right)$ be the class of Borel sets of additive class $\xi$ for the product of copies of the discrete topology on $X$ (the Polish topology on $X$ ), and let $\mathscr{B}=\cup_{\xi<\omega_{1}} \bar{\Sigma}_{\xi}$. We prove in the Lévy-Solovay model that

$$
\bar{\Sigma}_{\xi}=\Sigma_{\xi} \cap \mathscr{B}
$$

for $1 \leq \xi<\omega_{1}$.

Keywords. Borel sets of additive classes; Baire property; Levy-Solovay model; Gandy-Harrington topology.

\section{Introduction}

Suppose $X$ is a Polish space and $N$ the set of positive integers. We consider $H=X^{N}$ with two product topologies: (i) the product of copies of the Polish topology on $X$, so that $H$ is again a Polish space and (ii) the product of copies of the discrete topology on $X$. Define now the Borel hierarchy in the larger topology on $H$. To do so, we need some notation. An element of $H$ will be denoted by $h=\left(x_{1}, x_{2}, \ldots, x_{n}, \ldots\right)$ and for $m \in N, p_{m}(h)$ will denote the first $m$ coordinates, that is, $p_{m}(h)=\left(x_{1}, x_{2}, \ldots, x_{m}\right)$. For $n \in N$ and $A \subseteq X^{n}, \operatorname{cyl}(A)$ will denote the cylinder set with base $A$, that is,

$$
\operatorname{cyl}(A)=\left\{h \in H: p_{n}(h) \in A\right\} .
$$

The Borel hierarchy for the larger topology on $H$ can now be defined as follows:

$$
\Sigma_{0}=\Pi_{0}=\left\{\operatorname{cyl}(A): A \subseteq X^{n}, \quad n \geq 1\right\}
$$

and for $\xi>0$,

$$
\Sigma_{\xi}=\left(\bigcup_{\eta<\xi} \Pi_{\eta}\right)_{\sigma}, \quad \Pi_{\xi}=\neg \Sigma_{\xi} .
$$

The Borel hierarchy on $H$ with respect to the smaller topology is defined in the usual way:

$$
\bar{\Sigma}_{1}=\{V: V \text { is open in } H \text { in the smaller topology }\}, \bar{\Pi}_{1}=\neg \bar{\Sigma}_{1}
$$


and, for $\xi>1$,

$$
\bar{\Sigma}_{\xi}=\left(\bigcup_{\eta<\xi} \bar{\Pi}_{\eta}\right)_{\sigma} ; \bar{\Pi}_{\xi}=\neg \bar{\Sigma}_{\xi} .
$$

Let

$$
\mathscr{B}=\bigcup_{\xi<\omega_{1}} \bar{\Sigma}_{\xi}=\bigcup_{\xi<\omega_{1}} \bar{\Pi}_{\xi}
$$

The problem we will address in this article is whether

$$
\bar{\Sigma}_{\xi}=\Sigma_{\xi} \cap \mathscr{B} \quad \text { for } 1 \leq \xi<\omega_{1} .
$$

To tackle the problem we will use the methods of effective descriptive set theory. We therefore have to formulate the lightface version of $(*)$. We refer the reader to $[\mathrm{Mo}]$ and [L1] for definitions of lightface concepts. We take $X$ to be the recursively presentable Polish space $\omega^{\omega}$ hereafter.

Define

$$
\Sigma_{0}^{*}=\Pi_{0}^{*}=\left\{\operatorname{cyl}(A): A \text { is } \Delta_{1}^{1} \text { in }\left(\omega^{\omega}\right)^{n}, n \geq 1\right\},
$$

and, for $1 \leq \xi<\omega_{1}^{c k}$,

$$
\Sigma_{\xi}^{*}=\cup_{1}^{1}\left(\cup_{\eta<\xi} \Pi_{\eta}^{*}\right)
$$

and

$$
\Pi_{\xi}^{*}=\neg \Sigma_{\xi}^{*}
$$

where $\cup_{1}^{1}\left(\cup_{\eta<\xi} \Pi_{\eta}^{*}\right)$ is a $\Delta_{1}^{1}$ union of members of $\cup_{\eta<\xi} \Pi_{\eta}^{*}$. The lightface analogue of $(*)$ is then

$$
\Sigma_{\xi}^{*}=\Delta_{1}^{1} \cap \Sigma_{\xi}, \quad \text { for } 1 \leq \xi<\omega_{1}^{c k} .
$$

In order to state the main result of the article, we equip $\omega^{\omega}$ with the Gandy-Harrington topology, that is, the topology whose base is the pointclass of $\Sigma_{1}^{1}$ sets. The key property of this topology is that it satisfies the Baire category theorem (see [L1]). Consider now the following statement of set theory:

(O) Every subset of $\omega^{\omega}$ has the Baire property with respect to the Gandy-Harrington topology.

The main result of the article can now be stated.

Theorem 1.1. Assume (O). Let $1 \leq \xi<\omega_{1}^{c k}$. If $A$ and $B$ are $\Sigma_{1}^{1}$ subsets of $H$ such that $A$ can be separated from $B$ by a $\Sigma_{\xi}$ set, then $A$ can be separated from $B$ by $a \Sigma_{\xi}^{*}$ set.

An immediate consequence is

COROLLARY 1.2.

(O) implies $\left(^{* *}\right)$. 
The above results will be established in $\mathrm{ZF}+\mathrm{DC}$. Maitra et al $[\mathrm{Ma}]$ proved $\left(^{*}\right)$ for $\xi=1$ in $\mathrm{ZF}+\mathrm{DC}$ by a boldface argument. We will provide a lightface argument in the Appendix for $\left(^{* *}\right)$ when $\xi=1$. Again this will be done in $\mathrm{ZF}+\mathrm{DC}$. Barua [Ba] proved Theorem 1.1 and Corollary 1.2. His proof was by induction on $\xi$. However, he left out the proof of the base step $(\xi=1)$. We will fill in the gap in this article. The proof of Theorem 1.1 presented here parallels very closely that of Louveau [L1], whereas the proof in [Ba] relies on the more abstract developments of [L2]. In consequence, the proof given here is somewhat simpler.

The paper is organized as follows. Section 2 is devoted to definitions and notation. Section 3 contains the detailed proof of Theorem 1.1 when $\xi=1$, while $\S 4$ sketches how the proof of Theorem 1.1 can be completed by an inductive argument. In the concluding section, we will prove $\left(^{*}\right)$ under appropriate hypotheses and also mention open problems.

\section{Definitions, notation and preliminaries}

For $n \geq 1$, the Gandy-Harrington topology on $\left(\omega^{\omega}\right)^{n}$ will be denoted by $T^{n}$ and the Gandy-Harrington topology on $H$ will be denoted by $T^{\infty}$. Following Louveau [L1], we define for each $\xi$ such that $1 \leq \xi<\omega_{1}^{c k}$ a topology $T_{\xi}$ on $H$ having for its base the pointclass $\Sigma_{1}^{1} \cap \cup_{\eta<\xi} \Pi_{\eta}$.

Let $\mathscr{S}$ be a second countable topology on $\left(\omega^{\omega}\right)^{n}$ (respectively, $H$ ). Let $A$ be a subset of $\left(\omega^{\omega}\right)^{n}$ (respectively, $H$ ). By the cosurrogate of $A$ we mean the largest $\mathscr{S}$-open set $B$ such that $A \cap B$ is $T^{n}$-meager (respectively, $T^{\infty}$-meager). The surrogate of $A$ is defined to be the complement of the cosurrogate of $A$. When $\mathscr{S}$ is the topology $T^{n}$, we denote the surrogate (respectively, cosurrogate) of $A$ by $\operatorname{sur}^{n}(A)$ (respectively, $\left.\operatorname{cosur}^{n}(A)\right)$. If $A \subseteq H$ and $\mathscr{S}$ is the topology $T_{\xi}$, the surrogate (respectively, cosurrogate) of $A$ will be denoted by $\operatorname{sur}_{\xi}(A)$ (respectively, $\operatorname{cosur}_{\xi}(A)$ ).

Lemma 2.1. Let $m \geq 1$. If $A \subseteq\left(\omega^{\omega}\right)^{m}$ is $T^{m}$-open, then $\operatorname{sur}^{m}(A)$ is the $T^{m}$-closure of $A$. Consequently, $\operatorname{sur}^{m}(A)-A$ is $T^{m}$-nowhere dense.

Proof. If $B$ is $\Sigma_{1}^{1}$ and $A \cap B$ is $T^{m}$-meager, then $A \cap B$ must be empty, because $A \cap B$ is $T^{m}$ open and the Baire category theorem holds for $T^{m}$. Consequently, $\operatorname{cosur}^{m}(A)$ is the union of basic open sets of the $T^{m}$-topology which are disjoint with $A$. It follows that $\operatorname{sur}^{m}(A)$ is the $T^{m}$-closure of $A$.

Lemma 2.2. Assume (O). Let $m \geq 1$. If $A \subseteq\left(\omega^{\omega}\right)^{m}$, then $A \Delta \operatorname{sur}^{m}(A)$ is $T^{m}$-meager.

Proof. Observe that $\omega^{\omega}$ and $\left(\omega^{\omega}\right)^{m}$ are recursively isomorphic, so $\left(\omega^{\omega}, T^{1}\right)$ and $\left(\left(\omega^{\omega}\right)^{m}, T^{m}\right)$ are homeomorphic. Hence it follows from $(\mathbf{O})$ that there is a $T^{m}$-open set $B$ such that $A \Delta B$ is $T^{m}$-meager. So, if $D$ is a $\Sigma_{1}^{1}$ subset of $\left(\omega^{\omega}\right)^{m}$, then $A \cap D$ is $T^{m}$ meager iff $B \cap D$ is $T^{m}$-meager, so that $\operatorname{sur}^{m}(A)=\operatorname{sur}^{m}(B)$. Since $B$ is $T^{m}$-open, it follows from Lemma 2.1 that $\operatorname{sur}^{m} B-B$ is $T^{m}$-nowhere dense, hence $B \Delta \operatorname{sur}^{m}(B)$ is $T^{m}$ - meager. Consequently, $A \Delta \operatorname{sur}^{m}(A)$ is $T^{m}$-meager.

Note that the converse of Lemma 2.2 is true. Indeed, if $A \Delta \operatorname{sur}^{1}(A)$ is $T^{1}$-meager for every $A \subseteq \omega^{\omega}$, then, as is easy to verify, $A$ has the Baire property with respect to $T^{1}$ for every $A \subseteq \omega^{\omega}$, that is, $(\mathbf{O})$ holds.

\section{The case $\xi=1$}

In this section we will prove Theorem 1.1 when $\xi=1$. 
Following [L1], we fix a coding pair $(W, C)$ for the $\Delta_{1}^{1}$ subsets of $H$, that is,

(i) $W$ is a $\Pi_{1}^{1}$ subset of $\omega$;

(ii) $C$ is a $\Pi_{1}^{1}$ subset of $\omega \times H$;

(iii) the relations ' $n \in W \& C(n, h)$ ' and ' $n \in W \& \neg C(n, h)$ ' are both $\Pi_{1}^{1}$;

(iv) for every $\Delta_{1}^{1}$ subset $A$ of $H$, there is $n \in W$ such that $A=C_{n} \stackrel{\text { def. }}{=}\{h \in H: C(n, h)\}$.

Define $W_{0}$ as follows:

$$
\begin{aligned}
m & \in W_{0} \leftrightarrow m \in W \&(\exists n \geq 1)(\forall h)\left(\forall h^{\prime}\right)\left(C(n, h) \& p_{n}(h)\right. \\
& \left.=p_{n}\left(h^{\prime}\right) \rightarrow C\left(n, h^{\prime}\right)\right) .
\end{aligned}
$$

Then $W_{0}$ is $\Pi_{1}^{1}$. Indeed, $W_{0}$ is just the set of codes of $\Delta_{1}^{1}$ cylinder subsets of $H$.

Lemma 3.1. If $A$ is a $\Sigma_{1}^{1}$ subset of $H$, then $\mathrm{cl}_{1}(A)$ is $\Pi_{1}$ and $\Sigma_{1}^{1}$, hence $T_{2}$-open, where $\mathrm{cl}_{1}(A)$ is the $T_{1}$-closure of $A$.

Proof. Indeed, for any $A, \mathrm{cl}_{1}(A)$ is $\Pi_{1}$, because it is a countable intersection of $\Pi_{1}$ sets. Now suppose $A$ is $\Sigma_{1}^{1}$. Then

$$
\begin{aligned}
h \notin \operatorname{cl}_{1}(A) \leftrightarrow & (\exists n \geq 1)(\exists B)\left(B \text { is a } \Sigma_{1}^{1} \text { subset of }\left(\omega^{\omega}\right)^{n} \& h \in \operatorname{cyl}(B)\right. \\
& \& A \cap \operatorname{cyl}(B)=\phi) \\
\leftrightarrow & (\exists n \geq 1)(\exists B)\left(B \text { is a } \Delta_{1}^{1} \text { subset of }\left(\omega^{\omega}\right)^{n}\right. \\
& \& h \in \operatorname{cyl}(B) \& A \cap \operatorname{cyl}(B)=\phi) .
\end{aligned}
$$

To prove the previous implication $\rightarrow$, let $B$ be a $\Sigma_{1}^{1}$ subset of $\left(\omega^{\omega}\right)^{n}$ such that $h \in \operatorname{cyl}(B)$ and $A \cap \operatorname{cyl}(B)=\phi$. But then $p_{n}(A) \cap B=\phi$. Since $p_{n}(A)$ is $\Sigma_{1}^{1}$, it follows from Kleene's separation theorem that there is a $\Delta_{1}^{1}$ subset $B^{\prime}$ of $\left(\omega^{\omega}\right)^{n}$ such that $B \subseteq B^{\prime}$ and $B^{\prime} \cap p_{n}(A)=$ $\phi$. Hence $h \in \operatorname{cyl}\left(B^{\prime}\right)$ and $A \cap \operatorname{cyl}\left(B^{\prime}\right)=\phi$, which establishes $\rightarrow$. Consequently,

$$
h \notin \mathrm{cl}_{1}(A) \leftrightarrow(\exists m)\left(m \in W_{0} \& C(m, h) \& C_{m} \cap A=\phi\right) .
$$

So $\neg \mathrm{cl}_{1}(A)$ is $\Pi_{1}^{1}$.

Lemma 3.2. Assume (O). If $A$ is a $\Pi_{1}$ subset of $H$, then $A \Delta \operatorname{sur}_{1}(A)$ is $T^{\infty}$-meager.

Proof. Choose subsets $B_{n}$ of $\left(\omega^{\omega}\right)^{n}, n \geq 1$, such that

$$
A=H-\cup_{n \geq 1} \operatorname{cyl}\left(B_{n}\right) .
$$

Then

$$
\begin{aligned}
\operatorname{sur}_{1}(A)-A= & \operatorname{sur}_{1}(A) \cap \cup_{n \geq 1} \operatorname{cyl}\left(B_{n}\right) \\
\subseteq & \cup_{n \geq 1}\left(\left[\operatorname{sur}_{1}(A) \cap \operatorname{cyl}\left(\operatorname{sur}^{n}\left(B_{n}\right)\right)\right]\right. \\
& \left.\cup\left[\operatorname{cyl}\left(B_{n}\right)-\operatorname{cyl}\left(\operatorname{sur}^{n}\left(B_{n}\right)\right)\right]\right) .
\end{aligned}
$$

Now

$$
\operatorname{cyl}\left(B_{n}\right)-\operatorname{cyl}\left(\operatorname{sur}^{n}\left(B_{n}\right)\right)=\operatorname{cyl}\left(B_{n}-\operatorname{sur}^{n}\left(B_{n}\right)\right) .
$$


The set on the right of the above equality is $T^{\infty}$-meager by virtue of Lemma 2.13 in [L2]. We will now prove that $\operatorname{sur}_{1}(A) \cap \operatorname{cyl}\left(\operatorname{sur}^{n}\left(B_{n}\right)\right)$ is $T^{\infty}$-nowhere dense. Note that $\operatorname{sur}_{1}(A) \cap \operatorname{cyl}\left(\operatorname{sur}^{n}\left(B_{n}\right)\right)$ is $T_{1}$-closed, hence $T^{\infty}$-closed. Now let $A^{\prime}$ be a $\Sigma_{1}^{1}$ set contained in $\operatorname{sur}_{1}(A) \cap \operatorname{cyl}\left(\operatorname{sur}^{n}\left(B_{n}\right)\right)$. Then

$$
\operatorname{cyl}\left(p_{n}\left(A^{\prime}\right)\right) \subseteq \operatorname{cyl}\left(\operatorname{sur}^{n}\left(B_{n}\right)\right) .
$$

Hence

$$
\begin{aligned}
A \cap \operatorname{cyl}\left(p_{n}\left(A^{\prime}\right)\right) & \subseteq \operatorname{cyl}\left(\operatorname{sur}^{n}\left(B_{n}\right)\right)-\operatorname{cyl}\left(B_{n}\right) \\
& =\operatorname{cyl}\left(\operatorname{sur}^{n}\left(B_{n}\right)-B_{n}\right) .
\end{aligned}
$$

Consequently, by virtue of Lemma 2.2 and Lemma 2.13 in [L2], $A \cap \operatorname{cyl}\left(p_{n}\left(A^{\prime}\right)\right)$ is $T^{\infty}$ meager. Since cyl $\left(p_{n}\left(A^{\prime}\right)\right)$ is $T_{1}$-open, it follows that $\operatorname{cyl}\left(p_{n}\left(A^{\prime}\right)\right) \subseteq \operatorname{cosur}_{1}(A)$. Hence $A^{\prime}$ is empty because $A^{\prime}$ is also contained in $\operatorname{sur}_{1}(A)$. Thus $\operatorname{sur}_{1}(A) \cap \operatorname{cyl}\left(\operatorname{sur}^{n}\left(B_{n}\right)\right)$ is $T^{\infty}$ nowhere dense. It follow from (1) that $\operatorname{sur}_{1}(A)-A$ is $T^{\infty}$-meager. Since $A-\operatorname{sur}_{1}(A)$ is easily seen to be $T^{\infty}$-meager, we are done.

Lemma 3.3. If $A$ and $B$ are $\Sigma_{1}^{1}$ subsets of $H$ such that $A$ can be separated from $B$ by $a \Sigma_{1}$ set, then $A \cap \operatorname{cl}_{1}(B)=\phi$.

Proof. Suppose $D$ is a $\Pi_{1}$ subset of $H$ such that $A \cap D=\phi$ and $B \subseteq D$. Hence, by Lemma $3.2, B-\operatorname{sur}_{1}(D)$ is $T^{\infty}$-meager. But $B-\operatorname{sur}_{1}(D)$ is $T^{\infty}$-open, so $B \subseteq \operatorname{sur}_{1}(D)$.

Since $\operatorname{sur}_{1}(D)$ is $T_{1}$-closed, $\operatorname{cl}_{1}(B) \subseteq \operatorname{sur}_{1}(D)$. Now $A \cap \operatorname{sur}_{1}(D)$ is $T^{\infty}$-meager, so $A \cap$ $\mathrm{cl}_{1}(B)$ is $T^{\infty}$-meager. By Lemma 3.1, $A \cap \mathrm{cl}_{1}(B)$ is $\Sigma_{1}^{1}$, hence $A \cap \mathrm{cl}_{1}(B)$ must be empty.

Lemma 3.4. If $A$ and $B$ are $\Sigma_{1}^{1}$ subsets of $H$ such that $A \cap \operatorname{cl}_{1}(B)=\phi$, then $A$ can be separated from $B$ by a $\Sigma_{1}^{*}$ set.

Proof. Define

$$
P(h, n) \leftrightarrow h \notin A \vee\left(n \in W_{0} \& C(n, h) \& C_{n} \cap B=\phi\right) .
$$

Then $P$ is $\Pi_{1}^{1}$ and $(\forall h)(\exists n) P(h, n)$. By Kreisel's selection theorem [Mo], there is a $\Delta_{1}^{1}$ recursive function $f: H \rightarrow \omega$ such that $(\forall h) P(h, f(h))$. Let

$$
D=\left\{n \in \omega: n \in W_{0} \& C_{n} \cap B=\phi\right\} .
$$

Then $D$ is $\Pi_{1}^{1}$ and $f(A) \subseteq D$. Since $f(A)$ is $\Sigma_{1}^{1}$, there is a $\Delta_{1}^{1}$ set $E \subseteq \omega$ such that $f(A) \subseteq$ $E \subseteq D$. Let

$$
R(h, n) \leftrightarrow n \in E \& C(n, h),
$$

Then $R$ is $\Delta_{1}^{1}$, because if

$$
R^{\prime}(h, n) \leftrightarrow n \in E \& \neg C(n, h),
$$

then both $R$ and $R^{\prime}$ are $\Pi_{1}^{1}, R \cap R^{\prime}=\phi$ and $R \cup R^{\prime}=H \times E$. Set

$$
G_{n}=\{h: R(h, n)\}, n \in \omega .
$$

Then $\cup_{n \geq 0} G_{n}$ is a $\Sigma_{1}^{*}$ set which separates $A$ from $B$.

Lemmas 3.2, 3.3 and 3.4 establish Theorem 1.1 for $\xi=1$. 


\section{Proof of Theorem 1.1}

The proof of Theorem 1.1 is by induction on $\xi$. So we fix $\xi>1$ and assume Theorem 1.1 is true for all $\eta<\xi$. Lemmas 3.1-3.4 can be formulated and proved at level $\xi$, thereby completing the proof of Theorem 1.1 at level $\xi$. We omit the proofs because they are exactly like the proofs of Lemmas 7, 8, 9 and Theorem B in [L1].

We observe that the inductive hypothesis that Theorem 1.1 hold at all levels $\eta<\xi$ is by itself not sufficiently strong to prove the analogue of Lemma 3.2 at level $\xi$ and hence the theorem itself at that level. For this we need that analogues of Lemma 3.2 hold at all levels $\eta<\xi$. It is at this point in the proof that assumption $(\mathbf{O})$ is needed to ensure that Lemma 3.2 hold at level $\xi=1$, the higher levels of Lemma 3.2 then being proved by inducting up from the base level.

\section{Concluding remarks}

For $\alpha \in \omega^{\omega}$, we now consider the following statement of set theory:

$(\alpha)$ Every subset of $\omega^{\omega}$ has the Baire property with respect to the topology whose base is the pointclass of $\Sigma_{1}^{1}(\alpha)$ sets.

It is straightforward to relativize Theorem 1.1 to $\alpha$ under the assumption that $(\alpha)$ holds. The next result is provable in $\mathrm{ZF}+\mathrm{DC}+(\forall \alpha)((\alpha))$.

Theorem 5.1. Let $X$ be an uncountable Polish space and let $H=X^{N}$. Then, for $1 \leq \xi<$ $\omega_{1}$,

$$
\bar{\Sigma}_{\xi}=\Sigma_{\xi} \cap \mathscr{B} .
$$

Under the assumption that there is an inaccessible cardinal, Solovay [S] proved that $\mathrm{ZF}+\mathrm{DC}$ holds in the Lévy-Solovay model. Furthermore, it was observed by Louveau (p.43 of [L2]) that the statement $(\forall \alpha)((\alpha))$ holds as well in the model.

Whether Theorem 5.1 is provable in ZFC remains an open problem. Indeed, we do not have an answer to the problem even when $\xi=2$.

It is not difficult to prove that the axiom of determinacy implies $(\forall \alpha)((\alpha))$ so that Theorem 5.1 is provable in $\mathrm{ZF}+\mathrm{AD}$ (see [Mo]). On the other hand, the axiom of choice implies $\neg(\mathbf{O})$ in $\mathrm{ZF}$.

\section{Appendix}

We will now prove Theorem 1.1 for $\xi=1$ without assuming $(\mathbf{O})$. In view of Lemma 3.4, it will suffice to prove that $A \cap \operatorname{cl}_{1}(B)=\phi$. Define

$$
P(h, n) \leftrightarrow(n \geq 1) \&\left(\exists h^{\prime}\right)\left(p_{n}(h) h^{\prime} \in B\right),
$$

where $p_{n}(h) h^{\prime}$ is the catenation of $p_{n}(h)$ and $h^{\prime}$. Note that $P$ is $\Sigma_{1}^{1}$. Let

$$
h \in \bar{B} \leftrightarrow(\forall n \geq 1) P(h, n),
$$

so that $\bar{B}$ is the closure of $B$ in the product of discrete topologies on $H$. Consequently, $\bar{B} \subseteq H-A$. Define

$$
Q(h, n) \leftrightarrow(n \geq 1) \&(\neg P(h, n) \vee h \notin A) .
$$

Then $Q$ is clearly $\Pi_{1}^{1}$ and $(\exists n) Q(h, n)$. So there is a $\Delta_{1}^{1}$-recursive function $f: H \rightarrow \omega$ such that $(\forall h) Q(h, f(h))$. Let 


$$
S(h, n) \leftrightarrow(n \geq 1) \&(f(h) \neq n \vee h \notin A)
$$

\section{Claim.}

(i) $S$ is $\Pi_{1}^{1}$,

(ii) $(\forall h)(\forall n \geq 1)(P(h, n) \rightarrow S(h, n))$,

(iii) $h \notin A \leftrightarrow(\forall n \geq 1) S(h, n)$.

To see (ii), assume $P(h, n)$. Then we must have $h \in A \rightarrow f(h) \neq n$. Hence $S(h, n)$. For (iii), suppose $h \notin A$. Clearly, then $(\forall n \geq 1) S(h, n)$. Suppose now that $h \in A$. Then there is $n$ such that $f(h)=n$, hence $\neg S(h, n)$. (iii) now follows.

Now turn each $S_{n}$ into a cylinder set as follows. Define

$$
R(h, n) \leftrightarrow\left(\forall h^{\prime}\right) S\left(p_{n}(h) h^{\prime}, n\right),
$$

so $R$ is $\Pi_{1}^{1}$. Note that $P_{n}$ and $R_{n}$ are cylinder sets, that is,

$$
P(h, n) \& p_{n}(h)=p_{n}\left(h^{\prime}\right) \rightarrow P\left(h^{\prime}, n\right)
$$

and

$$
R(h, n) \& p_{n}(h)=p_{n}\left(h^{\prime}\right) \rightarrow R\left(h^{\prime}, n\right) .
$$

Claim. $(\forall h)(\forall n)(P(h, n) \rightarrow R(h, n))$.

So suppose $P(h, n)$. Then, for every $h^{\prime}, P\left(p_{n}(h) h^{\prime}, n\right)$, hence $S\left(p_{n}(h) h^{\prime}, n\right)$, so $R(h, n)$.

To complete the proof, let $h \in A$. Then there is $n \geq 1$ such that $\neg S(h, n)$, hence $\neg R(h, n)$. Now $\neg R_{n}$ is $\Sigma_{1}^{1}$ and $\Pi_{0}$ because $R_{n}$ is a cylinder set. Moreover, $\neg R_{n} \cap B=\phi$ because $\neg R_{n} \subseteq \neg P_{n}$ and $\neg P_{n} \cap B=\phi$. Hence $\neg R_{n}$ is a $T_{1}$-open set containing $h$ and disjoint from $B$. So $h \notin \mathrm{cl}_{1}(B)$.

\section{Acknowledgement}

The authors would like to thank the referee for making a number of helpful suggestions.

\section{References}

[Ba] Barua R, On Borel hierarchies of countable products of Polish spaces, Real Analysis Exchange 16 (1990-91) 60-66

[L1] Louveau A, A separation theorem for $\Sigma_{1}^{1}$ sets, Trans. Am. Math. Soc. 260 (1980) 363378

[L2] Louveau A, Ensembles analytiques et boréliens dans les espaces produits, Astérisque 78 (1980) 1-87

[Ma] Maitra A, Pestien V and Ramakrishnan S, Domination by Borel stopping times and some separation properties, Fund. Math. 135 (1990) 189-201

[Mo] Moschovakis Y N, Descriptive Set Theory (Amsterdam: North-Holland) (1980)

[S] Solovay R M, A model of set theory in which every set of reals is Lebesgue measurable, Ann. Math. 92 (1970) 1-56 